\title{
Front Matter: Volume 11321
}

, "Front Matter: Volume 11321," Proc. SPIE 11321, 2019 International Conference on Image and Video Processing, and Artificial Intelligence, 1132101 (27 November 2019); doi: 10.1117/12.2558300

SPIE Event: The Second International Conference on Image, Video Processing and Artifical Intelligence, 2019, Shanghai, China 


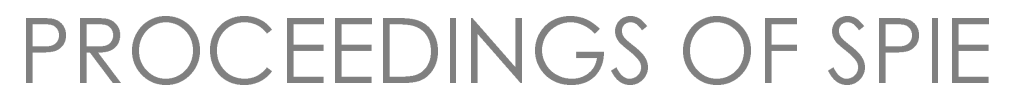

\section{International Conference on Image and Video Processing, and Artificial Intelligence}

\section{Ruidan Su}

Editor

\section{3-25 August 2019}

Shanghai, China

Organized by

Shanghai Advanced Research Institute, Chinese Academy of Sciences (China)

Published by

SPIE

Volume 11321 
The papers in this volume were part of the technical conference cited on the cover and title page. Papers were selected and subject to review by the editors and conference program committee. Some conference presentations may not be available for publication. Additional papers and presentation recordings may be available online in the SPIE Digital Library at SPIEDigitallibrary.org.

The papers reflect the work and thoughts of the authors and are published herein as submitted. The publisher is not responsible for the validity of the information or for any outcomes resulting from reliance thereon.

Please use the following format to cite material from these proceedings:

Author(s), "Title of Paper," in 2019 International Conference on Image and Video Processing, and Artificial Intelligence, edited by Ruidan Su, Proceedings of SPIE Vol. 11321 (SPIE, Bellingham, WA, 2019) Seven-digit Article CID Number.

ISSN: 0277-786X

ISSN: 1996-756X (electronic)

ISBN: 9781510634091

ISBN: 9781510634107 (electronic)

Published by

SPIE

P.O. Box 10, Bellingham, Washington 98227-0010 USA

Telephone +1 3606763290 (Pacific Time) · Fax + 13606471445

SPIE.org

Copyright @ 2019, Society of Photo-Optical Instrumentation Engineers.

Copying of material in this book for internal or personal use, or for the internal or personal use of specific clients, beyond the fair use provisions granted by the U.S. Copyright Law is authorized by SPIE subject to payment of copying fees. The Transactional Reporting Service base fee for this volume is $\$ 21.00$ per article (or portion thereof), which should be paid directly to the Copyright Clearance Center (CCC), 222 Rosewood Drive, Danvers, MA 01923. Payment may also be made electronically through CCC Online at copyright.com. Other copying for republication, resale, advertising or promotion, or any form of systematic or multiple reproduction of any material in this book is prohibited except with permission in writing from the publisher. The CCC fee code is 0277$786 \mathrm{X} / 19 / \$ 21.00$.

Printed in China.

Publication of record for individual papers is online in the SPIE Digital Library.

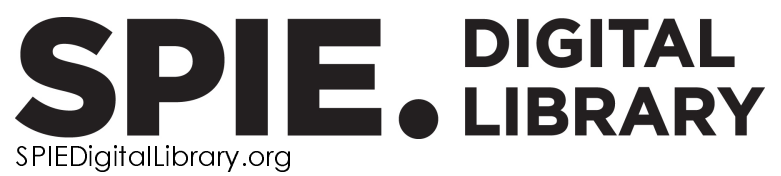

Paper Numbering: Proceedings of SPIE follow an e-First publication model. A unique citation identifier (CID) number is assigned to each article at the time of publication. Utilization of CIDs allows articles to be fully citable as soon as they are published online, and connects the same identifier to all online and print versions of the publication. SPIE uses a seven-digit CID article numbering system structured as follows:

- The first five digits correspond to the SPIE volume number.

- The last two digits indicate publication order within the volume using a Base 36 numbering system employing both numerals and letters. These two-number sets start with $00,01,02,03,04$, 05, 06, 07, 08, 09, OA, OB ... 0Z, followed by 10-1Z, 20-2Z, etc. The CID Number appears on each page of the manuscript. 


\title{
Contents
}

\author{
ix Authors \\ xiii Conference Committee \\ $\mathrm{xV} \quad$ Introduction
}

IMAGE PROCESSING AND APPLICATIONS

$1132102 \quad$ Automatic segmentation algorithm of license plate image based on PCNN and DNN [1 1321-7]

1132103 Adopting centrality measure models in visualized financial datasets [1 1321-9]

$1132104 \quad$ Functional magnetic resonance imaging classification based on random forest algorithm in Alzheimer's disease [1 $1321-11]$

$1132105 \quad$ Histogram-based image watermarking algorithm using visual perception characteristics [11321-14]

1132106 An offline fast model training method using CGAN for anti-jamming in true environment [11321-16]

1132107 The application of transfer learning for scene recognition [1 1321-18]

1132108 RGB-D dynamic facial dataset capture for visual speech recognition [1 1321-19]

$1132109 \quad$ Prototype of guide robot using marks in dynamic environments for visually impaired people [1 1321-20]

$113210 \mathrm{~A}$ The application of transfer learning in film and television works [11321-21]

$11321 \mathrm{OB} \quad$ Research on autonomous driving perception based on deep learning algorithm [11321-30]

$113210 \mathrm{C} \quad$ Asymmetric multiple-images cryptosystems based on discrete multiple-parameters fractional Fourier transforms [1 1321-34]

$113210 D \quad$ CNNs in the frequency domain for image super-resolution [1 1321-40]

$11321 \mathrm{OE} \quad$ Denoising and contrast enhancement fusion based on white balance for underwater images [1 1321-42]

11321 OF Pneumonia detection based on deep neural network Retinanet [1 1321-44] 
$113210 G \quad$ Human face detection based on skin color with principal component analysis (PCA) algorithm [1 $1321-50]$

$11321 \mathrm{OH} \quad$ A fractal image denoising algorithm in wavelet domain [1 1321-58]

$1132101 \quad$ A blind digital watermarking scheme based on RS coding [1 1321-59]

$113210 \mathrm{~J} \quad$ Walsh orthogonal moments for efficiently reducing Gibbs noise in image reconstruction [1 1321-62]

11321 OK Multi-loss joint optimization for person re-identification [1 1321-64]

11321 OL Feature optimization for pedestrian detection based on faster R-CNN [1 $1321-65]$

11321 OM Blind motion deblurring using multi-scale residual channel attention network [1 1321-70]

$113210 \mathrm{~N} \quad$ A texture segmentation method for high resolution remote sensing images combining gray edge information [11321-71]

$1132100 \quad$ Instance segmentation by using mask R-CNN based on feature fusion of RGB and depth images [1 1321-74]

11321 OP Real-time lane detection, fitting and navigation for unstructured environments [11321-92]

$113210 Q \quad$ Coordinated detection of robots during processing [1 1321-99]

$113210 R \quad$ Real-time patient facial expression recognition using convolutional neural network [11321-101]

11321 OS Laser-assisted turning spot detection algorithm for workpiece surface [11321-102]

11321 OT Night colorize: fully convolutional colorization network for low-light images [1 1321-107]

$113210 \mathrm{OU} \quad$ Lung image segmentation by generative adversarial networks [11321-118]

11321 OV Infrared and visible image fusion using joint convolution sparse coding [1 1321-122]

11321 OW Research on technology of image processing for ID photos [11321-123]

11321 OX Automatic recognition of radar signal types based on convolutional neural network [11321-124]

11321 OY Smooth voxel surface for medical volumetric rendering [11321-134]

$113210 Z$ Remote sensing image fusion based on dictionary learning and sparse representation [11321-142]

1132110 Transfer learning of deep CNN de-noiser prior for Chinese ancient calligraphy tablet image denoising [11321-144] 
$1132111 \quad$ Research and design of three-dimensional brain augmented reality system based on medical image features [1 1321-149]

\section{VIDEO PROCESSING AND APPLICATIONS}

1132112 A CAVE-based panoramic video playing method with $360^{\circ}$ viewpoint [1 1321-24]

1132113 Research on the application of modern information technology in teaching based on the network multimedia environment [1 1321-48]

1132114 Surveillance of abnormal behavior in elevators based on edge computing [1 1321-60]

$1132115 \quad$ Video action recognition based on improved 3D convolutional network and sparse representation classification [1 1321-73]

1132116 A fast blink-control system based on FPGA [11321-93]

$1132117 \quad$ Non-uniform correction of infrared image based on adaptive forgetting factor recursive least square method [1 1321-115]

1132118 SAR target recognition based on Gabor filter and convolutional neural network [11321-116]

1132119 Optimizing the function of urban intelligent transportation system [1 1321-128]

11321 1A Video-based traffic sign detection and recognition [1 1321-129]

\section{COMPUTER VISION AND AI}

11321 1B Design and implementation of encrypted file system based on FPGA [11321-15]

11321 1C Sheep delivery scene detection based on faster-RCNN [1 1321-22]

11321 ID A novel robot teaching system based on augmented reality [1 1321-39]

$113211 \mathrm{E} \quad$ Detection and fine 3D pose estimation of texture-less objects [1 1321-52]

11321 1F Joining geometric and RGB features for RGB-D semantic segmentation [1 1321-63]

$1132116 \quad$ XGAN: adversarial attacks with GAN [11321-82]

$113211 \mathrm{H} \quad$ Target tracking based on hierarchical feature fusion of residual neural network [1 1321-95]

$113211 \mathrm{~J} \quad$ MFM Net: modify feature map for object detection [11321-117] 
$113211 \mathrm{~K} \quad$ Receptive field enrichment network for pedestrian detection [1 1321-125]

$113211 \mathrm{~L} \quad$ Self-repairing object tracking method by adopting multi-level features [11321-126]

$113211 \mathrm{M} \quad$ Applying estimation models to accelerate genetic algorithms for charging scheduling problems in wireless rechargeable sensor networks [1 1321-133]

$113211 \mathrm{~N} \quad$ Efficient training and inference in highly temporal activity recognition [1 1321-147]

\section{MACHINE LEARNING AND ARTIFICIAL NEURAL NETWORKS}

$1132110 \quad$ Tai chi action recognition based on structural LSTM with attention module [11321-17]

11321 1P Research on intelligent internet financial investment model [11321-25]

$113211 Q \quad$ Pattern recognition by pattern inversion [1 1321-26]

$113211 \mathrm{R} \quad$ A research on generative adversarial network algorithm based on GPU parallel acceleration [1 1 $321-35]$

11321 is The classification of facial expressions with multi-cultural backgrounds: an event-related fMRI study [1 1321-37]

$113211 \mathrm{~T} \quad$ Prognostic recurrence analysis method for non-small cell lung cancer based on CT imaging [11321-41]

$113211 \mathrm{U}$ Task scheduling algorithm for non-unit time testing [1 1321-43]

11321 1V Community conflict prediction method based on spliced BiLSTM [1 1321-49]

11321 IW Research on path optimization of reverse logistics network [11321-53]

$113211 X \quad$ Entity relationship extraction optimization based on entity recognition [11321-66]

$113211 \mathrm{Y}$ Semantic-constraint graph dual non-negative matrix factorization in text co-clustering [1 1321-69]

$1132112 \quad$ Bagging deep autoencoders with dynamic threshold for semi-supervised anomaly detection [1 1321-75]

1132120 A research on cell inconsistency prediction of power battery using Gaussian process regression [1 1321-78]

1132121 A face reconstruction method based on fusion regression network and gradient descent [1 1321-79]

1132122 Research on face recognition technology and its application in intelligent security [11321-80] 
$1132123 \quad$ Ensemble learning based multi-source information fusion [1 $1321-81$ ]

$1132124 \quad$ Improved extreme learning machine and its application in SAR target recognition [11321-85]

1132125 Scene matching areas classification based on PCANet and MLP [1 1321-86]

1132126 Convolutional neural network with contextualized word embedding for text classification [1 1321-90]

1132127 An experimental verification for the convergence of the $\mathrm{BIBCI}$ algorithm [1 1321-91]

1132128 The visual synaesthesia analysis of Chinese traditional music aesthetics [1 1321-94]

1132129 Deep learning-based visual inspection for the delayed brittle fracture of high-strength bolts in long-span steel bridges [1 $1321-96]$

$113212 \mathrm{~A} \quad$ A new gait energy image based on mask processing for pedestrian gait recognition [11321-103]

$113212 \mathrm{~B} \quad$ Intelligent control method for high maneuvering fighter (Invited Paper) [11321-106]

$113212 \mathrm{C} \quad$ Auditory effect of chanting sound [11321-108]

$113212 \mathrm{D} \quad$ Convolutional neural networks application in cardiovascular decision support systems [11321-119]

$113212 \mathrm{E} \quad$ Analysis on EEG signal with machine learning [1 1321-120]

$113212 \mathrm{~F} \quad$ Development of upper limb rehabilitation training control system based on path planning [11321-127]

$113212 \mathrm{G}$ A novel improved CNN algorithm via denoising approach [1 1321-145]

$113212 \mathrm{H} \quad$ Research on textual classification of medical history in electronic patient records based on LSTM [1 1321-148]

\section{AI SYSTEMS}

$1132121 \quad$ A sparsity-relaxed algorithm for the under-determined convolutive blind source separation [1 1321-12]

$113212 \mathrm{~J} \quad$ Chinese dialect identification using prosodic classes and enhanced bigram model [1 1321-28]

$113212 \mathrm{~K} \quad$ An adaptive hierarchical multi-hop routing protocol based on energy balance in WSN [1 1321-31] 
$113212 \mathrm{~L} \quad$ WOA with adaptive mutation operator to estimate parameters of heavy oil thermal cracking model [1 1321-36]

$113212 \mathrm{M} \quad$ A new distance measure based on Pythagorean hesitant fuzzy sets and its application to multicriteria decision making [1 1321-57]

$113212 \mathrm{~N} \quad$ Video-based violence detection by human action analysis with neural network [1 1321-84]

1132120 Development of educational game based on Cocos2d-JS engine [1 1321-88]

$113212 \mathrm{P} \quad$ Research on autonomous maneuvering decision of UCAV based on approximate dynamic programming [11321-105]

$113212 Q \quad$ Research on vehicle control technology of brain-computer interface based on SSVEP [11321-131]

$113212 R \quad$ Quantization of deep convolutional networks [1 1321-132]

$113212 S$ Sentence-level sentiment analysis via BERT and BiGRU [1 1321-139]

$113212 T \quad$ IFPSS: intelligence fire point sensing systems in AloT environments [11321-141]

$113212 \mathrm{U} \quad$ Short-term solar PV forecasting based on recurrent neural network and clustering [1 1321-143]

BIG DATA AND LARGE-SCALE SCIENTIFIC COMPUTING

$113212 \mathrm{~V} \quad$ Collaborative adaptive scheduling scheme for multi-source big data tasks in the cloud [1 1321-4]

$113212 \mathrm{~W} \quad$ Hybrid recommendation algorithm based on logistic regression refinement sorting model [1 1321-29]

$113212 X \quad$ WiFi location method based on TSNE-KNN [11321-72]

$113212 Y \quad$ Summary on sensors in agricultural robots [11321-98]

$113212 Z$ Double threshold control genetic algorithm based on optimal protection [1 1321-109]

$1132130 \quad$ High-performance vehicle diagnostic information collection device for vehicle big data [1 1 $1321-112]$

$1132131 \quad$ Using electromagnetic parameters of targets and R language to enhance the accurate and rapid judgment [1 1321-113] 


\section{Authors}

Numbers in the index correspond to the last two digits of the seven-digit citation identifier (CID) article numbering system used in Proceedings of SPIE. The first five digits reflect the volume number. Base 36 numbering is employed for the last two digits and indicates the order of articles within the volume. Numbers start with 00, 01, 02, 03, 04, 05, 06, 07, 08, 09, OA, OB...0Z, followed by 10-1Z, 20-2Z, etc.

Agarwal, Siddhant, OP

Agrawal, Deepank, OP

Ahmed, Naveed, 08

Basarab, Mikhail, 2D

Cai, Jiaxin, OU

Cai, Siwen, 16

Cai, Xiaodong, $1 \mathrm{~V}$

Cao, Dong, $2 \mathrm{H}$

Cao, Guoxu, $1 \mathrm{G}$

Cao, Shuhua, $\mathrm{OZ}$

Cao, Zhiyuan, is

Cha, Jaehoon, 2E

Chai, Xurong, 06

Chakravarty, Debashish, OP

Chan, C. W., 2N

Chang, Shihui, 2W

Chang, Wei-Xuan, 30

Chao, Song, 20

Chao, Yuyan, 10

Chaowalit, Orawan, OY

Charkhabi, Masoud, $1 \mathrm{~N}$

Chen, Chen, OB

Chen, Chi-Chun, 30

Chen, Guannan, OE

Chen, Haibo, 1R, 22

Chen, Hong wei, $1 \mathrm{M}$

Chen, Jian, 24

Chen, Jingjing, $1 M$

Chen, Lina, OF

Chen, Pingping, 20

Chen, Si, IV

Chen, Xin, OR

Chen, Yen-Chiu, 2T

Chen, Yin-Tang, 30

Chen, Yong, 2B

Chen, Youguang, $1 Y$

Chen, Yuanyuan, 16

Cheng, Hongchang, OT

Chiang, Pai-Song, 31

Chien, Chiang-Ju, 31

Chuang, Ken H., $2 \mathrm{U}$

Cui, Delong, 2V

Cui, Jiangtao, OJ

Dai, Jiakai, OM

Deng, Xiangyu, 02

Domracheva, Anna, 2D

Dong, Jimei, is

Dong, Lingxiao, 10

Du, Wenwen, 05
Duan, Hui-hong, $1 T$

Fan, Gaoyang, 26

Fan, Tao, 1W

Fang, Xiaoyu, $1 \mathrm{G}$

Flores A., Christian E., 09

Fok, Wilton W. T., 2N

Fu, Qi, 15

Fu, Shilei, OR

Gan, Rui, $1 F$

Gang, Xiong, 20

Gao, Hong, $2 M$

Gao, Peng, 2P

Gao, Yan, 28

Ge, Feihang, 10

Geng, Xiaoran, 20

Guan, Zebiao, 1D

Guo, Aiying, 2G

Guo, Bingjun, $1 Z$

Guo, Chenlong, 17, 18

Guo, Kai, 2F

Guo, Xiuyan, 2M

Guo, Yi, 2I

Han, Shuai, 2K

Han, YuXuan, 18

$\mathrm{He}$, Bin, $2 \mathrm{Z}$

$\mathrm{He}$, Bing, OJ

He, Lifeng, 10

$\mathrm{He}$, Zhaorong, $1 \mathrm{x}$

Hong, Boyi, 07

Hong, Xin, 1D

Hou, Zhenzhen, $1 \mathrm{~V}$

Hsu, Huan-Po, 2T

Hsue, Wen-Liang, $0 \mathrm{C}$

Hu, Bowen, 1D

Hu, Jiwei, 14

Hu, Mengjie, $1 \mathrm{~L}$

$\mathrm{Hu}$, Zhencai, $2 \mathrm{P}$

Hua, Jiaxun, $1 Y$

Hua, Jie, 03

Huang, Lijie, $1 \mathrm{~S}$

Huang, Meifa, 2M

Huang, Min, OW

Huang, Sheng-Chi, OC

Huang, Yea-Shuan, 2R

Huo, Linsheng, 29

Ivanov, Igor, 2D

Jha, Arvind, OP

Ji, Ming, 23

Jiang, Chaohao, 1X 
Jiang, Minmin, 06

Jie, Xi, 2K

Jin, Chengxiong, 00

Jin, Cong, 07, OA

Jin, Hui, $1 \mathrm{H}$

Jin, Weiqi, OT, $1 \mathrm{~J}$

Jing, Xin, 11

Karavay, Mikhail, 1Q

Khachatryan, Michael, 2D

Khandelwal, Yash, OP

Kiaei, Dorsa S., OG

Kim, Kyeong Soo, 2E

Konnova, Natalia, 2D

Kowshik, Shreyas, OP

Lan, Hong, OW

Lan, Shanzhen, 10

Lee, Sanghyuk, 2E

Lei, Ming-Yuan, 2T

$\mathrm{Li}, \mathrm{AO}, 12$

Li, Bo, $1 \mathrm{~V}$

Li, Changsheng, 04

Li, Da-peng, 06

Li, Dongmei, 10

Li, Hongnan, 29

Li, Le, 23

Li, Li, OT, $1 \mathrm{~J}$

Li, Peng, $2 Y$

Li, Qiuni, 2B

Li, Shaobin, 10

Li, Shundong, 05

Li, Wei, $1 \mathrm{U}$

$\mathrm{Li}$, Xiaoyang, $1 \mathrm{~L}$

$\mathrm{Li}$, XinYang, $1 \mathrm{H}$

Li, Yajie, 07, OA

Li, Yaran, 2K

Li, Yuqing, 1D

Li, Zhong, 2A

Lian, Bihan, OA

Liang, Haoran, $1 \mathrm{Z}$

Liang, Xining, 17

Liang, Yi-Hua, 30

Liao, Xiaodong, 25

Ling, Liu, 20

Liu, Bin, $2 \mathrm{~F}$

Liu, Chung-Hsing, 2T

Liu, Chunyu, is

Liu, Hualing, $1 \mathrm{P}$

Liu, Jingjing, $2 G$

Liu, Jinlei, $2 Y$

Liu, Jun-Yu, OC

Liu, Lei, 22

Liu, Mao, OF

Liu, Qiujie, 19

Liv, Wang, 15

Liv, Xiangpeng, $2 Y$

Liu, Yadong, 2Q

Liu, Ya-li, 2C

Liu, Yi, 1D

LiU, Yingnan, OD

Liu, Yixuan, 17
Liu, Yongfeng, $2 F$

Liv, Yu, $1 Y$

Liu, Zongcheng, 2B

Lodhi, Vaibhav, OP

Long, Sheng, $2 Q$

Lou, Ping, 14

LU, Shuhua, OK, OL

Lu, Yuqiu, 15

Luo, Pengfei, $1 \mathrm{~K}$

Luo, Xiaonan, 1X, 2M, 2X

Ma, Shiwei, 00, 15

Ma, Xinyu, 28

Mikhailov, Alexei, 1Q

Mohta, Vibhakar, OP

Mu, Fuqi, 06

Nie, Sheng-dong, $1 T$

Ning, Wei-lin, 2C

Ouyang, Wen, $2 \mathrm{U}$

Ouyang, Zhiyou, $1 \mathrm{G}$

Paffenroth, Randy Clinton, OD

Pan, Liang, 25

Pang, Yufei, 11

Peng, Jian, $1 \mathrm{E}$

Peng, Xiaofei, 13

Peng, Yanguo, 0J

Pensiri, Fuangfar, OY

Pérez C., Aníbal R., 09

Qi, Yan, 14

Qi, Yunping, 02

Qian, Yutong, OR

Qin, Jinhui, 1J

Qin, Junping, 1C

Qin, Wenjuan, 02

Qin, Yuchu, 2M

Qiu, SU, OT, 1J

Qiu, Xin, 06

Rahurkar, Nivedita, $1 \mathrm{~N}$

Ren, Chunxiao, 27

Ren, Mengxue, OK, OL

Ren, Yan, 11

Romero S., Jhonny M., 09

Ruan, Guoging, OX

Rumipamba L., José A., 09

Shen, Jianghong, $2 S$

Shen, Yongliang, 1A

Shrivastava, Shrey, OP

Singhal, Apoorve, OP

Slot, Charles Djimy, 2R

Sodsong, Nattawat, $2 \mathrm{U}$

Song, Gangbing, 29

Song, Huapeng, $1 \mathrm{G}$

Song, Lei, $1 Z$

Song, Qian, OR

Song, Sutao, is

Su, Jianyong, 20

Sun, Jinyu, 00, 15

Sun, Kai, 25

Sun, Linjia, 2J

Sun, Shiwen, 1C

Sun, Ying, IW 
Sun, Zhihao, 06

Tai, Yu, OC

Tan, Dingying, 20

Tan, Yumeng, OF

Tang, Jing, 1R, 22

Tao, Jia, $1 \mathrm{R}$

Tao, Zhuang, $2 S$

Tavakoli, Saeed, OG

Tsai, Nancy, 2T

Visutsak, Porawat, oY

Wang, Daoshun, 05

Wang, Donghui, 11

Wang, Fei, 2P

Wang, Guohua, 03

Wang, Hongfei, $1 \mathrm{Z}$

Wang, Hongliang, 07, 0A

Wang, Junfeng, $\mathrm{OE}$

Wang, Mingyu, $O Q$

Wang, Mingyu, 2G

Wang, $\mathrm{Na}, \mathrm{OH}, \mathrm{Ol}, \mathrm{ON}$

Wang, Nansu, 07, OA

Wang, Ning, 2L

Wang, Peng, 16

Wang, Pengcheng, 10

Wang, San-Fu, 30

Wang, Wen-ming, 20

Wang, Xiaoyuan, 1B

Wang, $\mathrm{XU}, 1 \mathrm{~T}$

Wang, Yan, 2K

Wang, $Y u, 04$

Wang, $Y u, 2 Z$

Wang, Zengfu, $1 \mathrm{~K}$

Wei, Chao, OE

Wei, Shuhua, is

Wei, Wenguo, 2W

Wu, Haimei, $2 \mathrm{H}$

Wu, Wei, OX

WU, Xuefeng, OQ, OS

Wu, Yuxiao, 27

Wu, Zhiqiang, $2 Z$

$\mathrm{Xi}, \mathrm{Li}, 20$

Xia, Lubin, OT

Xiao, Yuyao, 2G

Xie, Guiyuan, 2W

Xie, Lingyun, 28

Xie, Qingbo, $2 X$

Xie, Yong-bo, 20

Xiong, Jiulong, 2A

Xiong, Ying, $1 \mathrm{~L}$

$\mathrm{Xu}, \mathrm{Chi}, 1 \mathrm{D}$

$X U$, Dantong, 11

$\mathrm{Xu}$, Junyi, 23

$\mathrm{Xu}, \mathrm{Xiaojie,} \mathrm{OZ}$

$\mathrm{Xu}$, Youquan, 03

Xue, Hongcheng, $1 \mathrm{C}$

Yan, Dan, OV

Yan, Junwei, 14

Yan, Ming, 21

Yang, Chao, 21

Yang, Chengfu, 19
Yang, Cheng-Wei, 30

Yang, Hongbo, $2 F$

Yang, Junjie, 21

Yang, Wanmeng, 1P

Yang, Xingchun, OV

Yang, Zhongming, $1 \mathrm{U}$

Yang, Zuyuan, 2l

Ye, Hui, 2H

Ye, Long, 12

Ye, Xiangbin, 2A

Yi, Zhang, 1A

Yin, Fei, $O Z$

Yin, Pei, OB

YU, Aiping, 11

Yu, Chang WU, 1M, 2R

YU, Kun-Ming, 2T, 2U

Yu, Yang, 2Q

Yuan, Weilin, 25

Yue, Zhen, OV

Zeng, Gang, $1 \mathrm{~F}$

Zeng, Wenquan, IU

Zeng, Yujun, OM

Zhai, Yang, OB

Zhang, Chengfang, OV

Zhang, Haolan, 2E

Zhang, HuiYing, 18

Zhang, Jiacai, 15

Zhang, Jie, 2G

Zhang, Nannan, $2 Q$

Zhang, Qiang, 16

Zhang, Qiuyu, 1A

Zhang, Ran, 02

Zhang, Shaopeng, $1 F$

Zhang, Shuyve, 2L

Zhang, Wei, $2 Y$

Zhang, Wenqian, 2B

Zhang, Yingbo, $1 \mathrm{E}$

Zhang, Yuxing, $2 Z$

Zhao, Leixian, $1 \mathrm{X}$

Zhao, Menglin, 21

Zhao, Shasha, $2 \mathrm{~F}$

Zhao, Shuaijie, $2 X$

Zhao, Wang, 11

Zhao, Xianghui, 05

Zhao, Ying, 11

Zhao, Yunqing, $2 \mathrm{~N}$

Zheng, Jihu, OB

Zheng, Lizi, 2V

Zheng, Taisheng, $1 \mathrm{Z}$

Zhong, Ming, $1 \mathrm{~F}$

Zhong, Yanru, 1X, 2M, 2X

Zhou, Bolin, OB

Zhou, Jing, 29

Zhou, Saijun, $1 \mathrm{P}$

Zhou, Shaojun, 1E

Zhou, Xianliang, OS

Zhou, Zongtan, $2 Q$

Zhu, Cui, 26

Zhu, Hongfeng, OU

Zhu, Lihua, 2 Z 
Zhu, Lin, 2K

Zhu, Wenjun, 26

Zhuo, Yirong, $2 \mathrm{H}$

Zou, Min, 20

Zuo, Renwei, 2B

Proc. of SPIE Vol. 11321 1132101-12

Downloaded From: https://www.spiedigitallibrary.org/conference-proceedings-of-spie on 26 Apr 2023 Terms of Use: https://www.spiedigitallibrary.org/terms-of-use 


\title{
Conference Committee
}

\author{
Conference Chair
}

Huaiyu Xu, Laboratory of Software Engineering, Shanghai Advanced Research Institute, Chinese Academy of Sciences (China)

Program Chairs

Ruidan Su, Laboratory of Software Engineering, Shanghai Advanced Research Institute, Chinese Academy of Sciences (China)

Shyi-Ming Chen, National Taiwan University of Science and Technology (Taiwan, China)

Han Liu, Cardiff University (United Kingdom)

Program Committee

Hao Ying, Wayne State University (United States)

Young-Long Chen, National Taichung University of Science and Technology (Taiwan, China)

Shou-Hsiung Cheng, Chienkuo Technology University (Taiwan, China)

Kun-Ming Yu, Chung Hua University (Taiwan, China)

Jyh-Cheng Chen, National Yang-Ming University (Taiwan, China)

Huei-Wen Ferng, National Taiwan University of Science and Technology (Taiwan, China)

Tan Tse Guan, Universiti Malaysia Kelantan (Malaysia)

Chang-Wu Yu, Chung Hua University (Taiwan, China)

Wong Kin Hong, Chinese University of Hong Kong (Hong Kong, China)

S. Kanthalakshmi, PSG College of Technology (India)

Ireneusz Kubiak, Military Communication Institute (Poland)

Hyo Jong Lee, Chonbuk National University (Korea, Republic of)

Xuefeng Liang, Kyoto University (Japan)

Shin-Feng Lin, National Dong Hwa University (Taiwan, China)

Hsiang-Chuan Liu, National Taichung University of Education (Taiwan, China)

Amir Nasrollahi, University of Pittsburgh (United States)

Xiaolin Qin, University of Chinese Academy of Sciences (China)

Rosalina Abdul Salam, Universiti Sains Islam Malaysia (Malaysia)

Álvaro Suárez Sarmiento, Universidad de las Palmas de Gran Canaria (Spain)

Victor R. L. Shen, National Taipei University (Taiwan, China)

Tawei David Shou, University of Taipei (Taiwan, China)

Santiago Pérez Suárez, Universidad de Las Palmas de Gran Canaria (Spain)

Tsai-Fong Tan, Ching Kuo Institute of Management \& Health (Taiwan, China) 
Philippe Thomas, Université de Lorraine (France)

Sinnu Susan Thomas, Indian Institute of Technology, Kanpur (India)

Birjodh Tiwana, Linkedin, Inc. (United States)

Rubén Usamentiaga, Universidad de Oviedo (Spain)

Cheng-Yi Wang, Tamkang University (Taiwan, China)

W. W. Yan, SARI, Chinese Academy of Sciences (China)

Xin-Yao Zou, Guangdong AIB Polytechnic College (China)

Mohammad (Behdad) Jamshidi, Islamic Azad University (Iran)

Yassine Al-Amrani, Abdelmalek Essaâdi University (Morocco)

Shlok Gilda, SimpleToken (India)

Mohammed Abdallah Bakr Mahmoud, Beijing Institute of Technology (China)

P. K. Verma, Rajkiya Engineering College (India)

Shamneesh Sharma, Alakh Prakash Goyal (APG) Shimla University (India)

Sivaramakrishnan Rajaraman, National Library of Medicine, National Institutes of Health (United States)

Sankhanil Dey, Calcutta University (India)

Ivanna Dronyuk, Lviv Polytechnic National University (Ukraine)

Ivan Inzonin, Lviv Polytechnic National University (Ukraine)

Majida Laaziri, Abdelmalek Essaâdi University (Morocco)

Benmoussa Khaoula, Abdelmalek Essaâdi University (Morocco)

P. M. K. Prasad, GVP College of Engineering for Women (India)

Alejandro Medina Santiago, Instituto Nacional de Astrofísica, Óptica y Electrónica (Mexico)

Gunasekar Thangarasu, Linton University College, Mantin (Malaysia)

Issa Atoum, The World Islamic Sciences \& Education University (Jordan)

Rajmohan Pardeshi, KRE's Karnatak Arts Science and Commerce College (India)

Shiba P. Kuanar, University of Texas, Arlington (United States)

Aruna Kumar S. V., Visvesvaraya Technological University, Belagavi (India)

Vinh Truong Hoang Ruong, Ho Chi Minh City Open University (Viet Nam) 


\section{Introduction}

The 2019 2nd International Conference on Image, Video Processing and Artificial Intelligence (IVPAI 2019) provided a forum for the most up-to-date and authoritative knowledge from both the industrial and academic worlds, sharing the newest research fruits and experiences in this exciting field. The second IVPAI was held in Shanghai, China during 23-25 August 2019.

It was organized by Shanghai Advanced Research Institute, Chinese Academy of Sciences, Shanghai, China. IVPAI 2019 is to bring together innovative academics and industrial experts in the field of image, video processing and artificial intelligence to a common forum.

The scientific program for the event was filled with presentations delivered by researchers and scholars from the international community, including keynote speeches and highly selective lectures. IVPAI received a total of 150 submitted papers this year. This volume contains 108 selected papers, which were presented at the conference. Every paper in this collection underwent rigorous peer-review performed by the conference technical committee and international reviewers before being selected for publication.

We hope that the contents of this volume prove useful for researchers and practitioners working in the fields of image processing and artificial intelligence in their efforts to develop and apply new technologies and processes.

The organizing committee express sincere gratitude to all the authors who presented their works at IVPAI 2019 and contributed in this way to the success of this event. Special thanks are due to the authors from aboard for attending the conference and to the reviewers for their great support in improving the quality of the papers in this volume. The organizing committee also address warmest thanks to all the members of the international committee for their support and the many others for their contribution in organizing IVPAI 2019.

Ruidan Su 
Proc. of SPIE Vol. 11321 1132101-16

Downloaded From: https://www.spiedigitallibrary.org/conference-proceedings-of-spie on 26 Apr 2023 Terms of Use: https://www.spiedigitallibrary.org/terms-of-use 